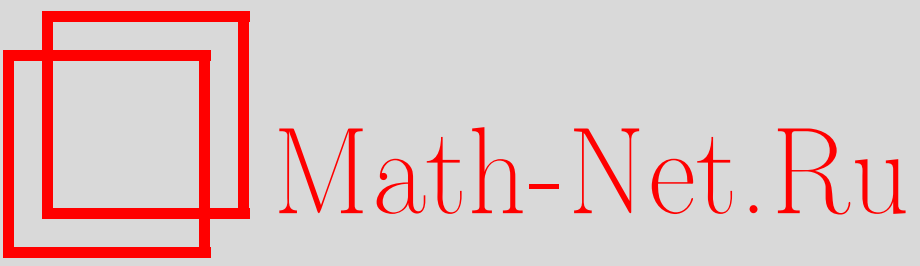

В. И. Арнольд, Геометрия и динамика полей Галуа, УМН, 2004, том 59, выпуск $6,23-40$

DOI: https://doi.org/10.4213/rm793

Использование Общероссийского математического портала Math-Net.Ru подразумевает, что вы прочитали и согласны с пользовательским соглашением

http://www.mathnet.ru/rus/agreement

Параметры загрузки:

IP : 3.85 .183 .62

26 апреля 2023 г., $16: 25: 36$ 


\title{
ГЕОМЕТРИЯ И ДИНАМИКА ПОЛЕЙ ГАЛУА
}

\author{
В. И. Арнольд
}

\begin{abstract}
Таблицы, задающие операции в конечных полях, обладают многими свойствами таблиц случайных чисел. Речь идет о своеобразном варианте автоморфизмов торов теории динамических систем, в котором число точек тора конечно.

Устанавливаются также действия преобразований Фробениуса конечных полей на проективные структуры конечных проективных пространств, описывающих геометрию поля.

Библиография: 1 название.
\end{abstract}

\section{СОДЕРЖАНИЕ}

$\S 1$. Построение таблицы поля .................................... 23

$\S 2$. Эргодические свойства таблиц полей Галуа $\quad \ldots \ldots \ldots \ldots \ldots \ldots \ldots \ldots \ldots . . . \ldots . . .27$

А) Гипотеза о равномерном распределении по площади таблицы . . . . . . . . . 27

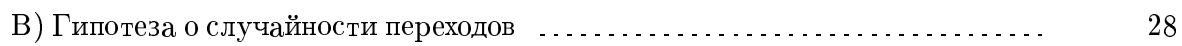

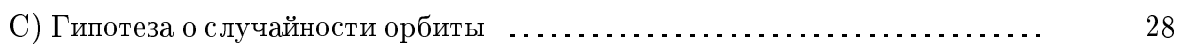

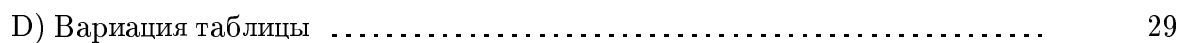

$\S 3$. Проективное многообразие поля Галуа .......................... 29

$\S 4$. Преобразования Фробениуса и их проективизации $\ldots . \ldots \ldots \ldots \ldots . . . . . . . .232$

$\S 5$. Проективные структуры поля Галуа из $p^{2}$ элементов, где $p=11 \quad \ldots \ldots . \quad 35$

$\S 6$. Проективные структуры поля Галуа из $p^{2}$ элементов, где $p=13 \ldots \ldots . \quad 38$

Список литературы .................................................... 40

\section{§1. Построение таблицы поля}

ОПРЕДЕЛЕНИЕ. Полем Галуа называется поле из конечного числа элементов.

Простейшим примером является поле $\mathbb{Z}_{p}=\mathbb{Z} /(p \mathbb{Z})$ вычетов по простому модулю $p$. Оно состоит из $p$ элементов.

Работа выполнена при частичной поддержке Российского фонда фундаментальных исследований (грант № 02-01-00655). 
Классическая теорема об этих полях такова:

ТеОРема. Число элементов любого поля Галуа равно $p^{a}$, где $p$ - простое число, а - натуральное число. Поле из такого числа әлементов существует (для любого числа $p^{a}$ ), и оно единственно ( $с$ точностью, конечно, до изоморфизма полей).

Чтобы записать операции сложения и умножения в поле, перенумеруем его элементы следуюшим образом. В поле есть элемент нуль, 0 (определяемый однозначно условием “ $0+x=x$ для любого элемента $x$ ”), а все остальные элементы образуют группу ("мультипликативную группу $\mathscr{F} \backslash 0$ поля $\mathscr{F} ")$ по умножению, т.е. имеют обратные элементы $x^{-1}$, для которых $x x^{-1}=1$ (где элемент 1 однозначно определяется условием “ $y 1=y$ для любого элемента $y$ ”).

Оказьвается, мультипликативная група поля - всегда циклическая группа (порядка $p^{a}-1$ для поля из $p^{a}$ элементов), т.е. ее элементы можно выразить через один из них, $A$ ( “мультипликативную образуюшую”), как

$$
A, A^{2}, A^{3}, \ldots, A^{p^{a}-1}=1 .
$$

Образуюшая $A$ может быть выбрана по-разному. А именно, за образующую можно взять любой элемент $B=A^{k}$, для которого $k$ взаимно просто с $p^{a}-1$.

Таким образом, число мультипликативных образуюших равно значению функции Эйлера, $\varphi\left(p^{a}-1\right)$.

ПримеР. Для поля из $p^{a}=49$ элементов число образуюших есть $\varphi(48)=16$, а именно, образующими являются элементы $A^{k}$, где

$$
k=1,5,7,11,13,17,19,23,25,29,31,35,37,41,43,47 .
$$

Все мультипликативные образующие образуют подгруппу по умножению (в кольце вычетов по модулю $\left.p^{a}-1\right)$, например, для $p^{a}=49$ :

$$
5 \cdot 5=25, \quad 7 \cdot 7=1, \quad 5 \cdot 11=7 .
$$

По сложению поле из $p^{a}$ элементов представляет собой $p$-векторное пространство $\left(\right.$ коммутативную групш $\left.\left(\mathbb{Z}_{p}\right)^{a}\right)$, так что его $p^{a}$ элементов можно записать в клеточки “конечного тора" размерности $a$,

$$
x=x_{1} e_{1}+\cdots+x_{a} e_{a},
$$

где $\left(e_{1}, \ldots, e_{a}\right)$ - аддитивные образующие, а коэффициенты $x_{j}$ - вычеты по модулю $p$.

ПримеР. Поле из 49 элементов состоит из комбинаций

$$
x=\alpha 1+\beta A
$$

где 1 и $A$ - элемент “единица" поля и мультипликативная образуюшая, обсужденная вьше (в общем случае в качестве аддитивных образуюших можно взять элементы $\left.\left\{A, A^{2}, \ldots, A^{a-1}, A^{a}=1\right\}\right)$. 
Для поля из $p^{2}$ элементов мы заключаем, что

$$
A^{2}=\alpha 1+\beta A
$$

с некоторыми коэффициентами $\alpha$ и $\beta$ из $\mathbb{Z}_{p}$.

Из соотношения (1) легко вьвести формулы для всех элементов поля:

$$
A^{3}=A \cdot A^{2}=\alpha A+\beta A^{2}=\alpha A+\beta(\alpha 1+\beta A)=p_{3} 1+q_{3} A,
$$

где $p_{3}=\alpha \beta, q_{3}=\alpha+\beta^{2}$.

Точно так же находим

$$
A^{k}=p_{k} 1+q_{k} A, \quad\left(p_{k}=\alpha q_{k-1}, \quad q_{k}=\beta q_{k-1}+p_{k-1}\right) .
$$

Эти рекуррентные соотношения позволяют легко вьписать многочлены $p_{k}$ и $q_{k}$ от $\alpha$ и $\beta$, коэффициенты которых - числа сочетаний. Многочлен $p_{k}$ имеет степень $2 k$, a многочлен $q_{k}$ - степень $2 k-1$, если считать, что степень $\alpha$ есть 2 , а степень $\beta$ есть 1 , как подсказьвает соотношение (1).

Явные формулы для коэффициентов многочленов $p_{k}$ и $q_{k}$ получаются вьписьванием ответов при $k=4,5,6$ :

$$
\begin{array}{ll}
p_{4}=\alpha^{2}+\alpha \beta^{2}, & q_{4}=2 \alpha \beta+\beta^{3} ; \\
p_{5}=2 \alpha^{2} \beta+\alpha \beta^{3}, & q_{5}=\alpha^{2}+3 \alpha \beta^{2}+\beta^{4} ; \\
p_{6}=\alpha^{3}+3 \alpha^{2} \beta^{2}+\alpha \beta^{4}, & q_{6}=3 \alpha^{2} \beta+4 \alpha \beta^{3}+\beta^{5},
\end{array}
$$

и их сравнением с треугольником Паскаля:

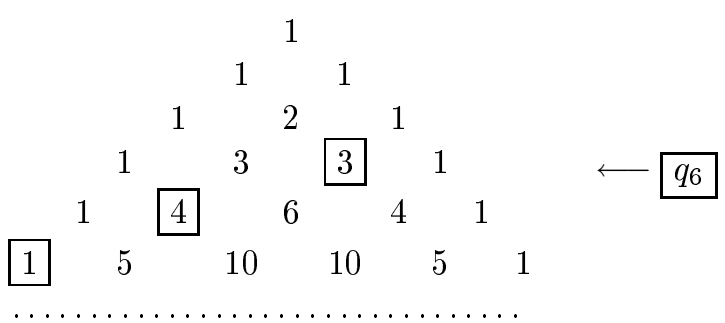

Выделены коэффициенты многочлена $q_{6}$ : они соответствуют биномиальньг членам степени 5 при $\operatorname{deg} \beta=1, \operatorname{deg} \alpha=2$, т.е. член $\alpha^{u} \beta^{v}$ входит в $q_{6}$ при $2 u+v=5$ со своим биномиальным коэффициентом $C_{u+v}^{v}$.

Чтобы выбрать образуюшую $A$, нужно подобрать коэффициенты $\alpha$ и $\beta$ (вычеты по модулю $p$ ) так, чтобы

$$
A^{k}=1 \text { при } k=p^{2}-1, \quad A^{k} \neq 1 \text { при } k<p^{2}-1 .
$$

Эти сравнения

$$
\left[\left\{\begin{array}{l}
p_{k}(\alpha, \beta)=1 \text { при } k=p^{2}-1, \quad\left(p_{k}, q_{k}\right) \neq(1,0) \text { при } k<p^{2}-1 \\
q_{k}(\alpha, \beta)=0
\end{array}\right]\right.
$$

легко решаются при конкретных $p^{2}$. В случае $p^{a}$ элементов поля рассуждения аналогичны, хотя в (1) вместо $p^{2}$ нужно рассматривать $p^{a}$ (и вместо $A^{2}$ разлагать $A^{a}$ ).

После этого можно реализовать поле, считая уравнение (1) характеристическим для матрищы $A$, т.е. (при $a=2$ ) выбирая в качестве $A$ матрицу порядка 2 со следом $\beta$ и определителем $-\alpha$, например, $(A)=\left(\begin{array}{ll}0 & \alpha \\ 1 & \beta\end{array}\right)$. 
ПримеР. Для $p^{a}=49$ допустим выбор $\alpha=\beta=2,(A)=\left(\begin{array}{ll}0 & 2 \\ 1 & 2\end{array}\right)$. Поле состоит из 49 матриц: 0 и $A^{k}, k=1, \ldots, 48$, причем $A^{48}=1$.

ЗАмечание. Смысл матрищы $A$ состоит в следующем. Оператор умножения всех элементов поля на элемент $A$ является линейным оператором $\left(\mathbb{Z}_{p}\right)^{a} \rightarrow\left(\mathbb{Z}_{p}\right)^{a}$ в векторном пространстве $\left(\mathbb{Z}_{p}\right)^{a}$ (в примере $a=2-$ на плоскости из $p^{2}$ точек).

Базис этой плоскости образуют векторы $1=1 e_{1}+0 e_{2}$ и $A=0 e_{1}+1 e_{2}$ (где $e_{1}-$ это элемент 1 поля, а $e_{2}-$ элемент $A$; элемент выделен для того, чтобы не спутать его с коэффициентом 1 , принадлежашим $\mathbb{Z}_{p}$ ).

Оператор, переводящий любой элемент $x$ поля в элемент $A x$, линеен и имеет матрицей $(A)=\left(\begin{array}{ll}0 & \alpha \\ 1 & \beta\end{array}\right)$, так как $A\left[1=A, A\left[A=A^{2}=\alpha 1+\beta A\right.\right.$, т.е. $(A)\left(\begin{array}{l}1 \\ 0\end{array}\right)=\left(\begin{array}{l}0 \\ 1\end{array}\right)$, (A) $\left(\begin{array}{l}0 \\ 1\end{array}\right)=\left(\begin{array}{l}\alpha \\ \beta\end{array}\right)$.

Выбрав таким образом обозначения, мы можем заполнить последовательно каждую клеточку конечного “тора" $\left(\mathbb{Z}_{p}\right)^{2}$ стояшим в ней элементом поля: элемент

$$
A^{k}=p_{k} 1+q_{k} A
$$

записьвается в клетке с координатами $\left(p_{k}, q_{k}\right)$ в виде своего номера $k$, последовательно, при $k=1,2, \ldots,\left(p^{2}-1\right)$ (начиная с $A^{1}=A$ в клетке $(0,1)$ и кончая $A^{p^{2}-1}=1$ в клетке $(1,0)$ ). Результат назьвается таблицей поля (для поля из $p^{a}$ элементов таблица заполняет $a$-мерньй куб над $\left.\mathbb{Z}_{p}\right)$.

ПримеР. Для $p^{a}=49$ эти вычисления дают таблищу

\begin{tabular}{|c|c|c|c|c|c|c|}
\hline 25 & $25 \quad 44$ & 7 & 30 & 3 & 45 & 42 \\
\hline $5 A$ & 35 & 28 & 29 & 39 & 26 & 14 \\
\hline 17 & $17 \quad 37$ & 22 & 36 & 34 & 43 & 47 \\
\hline 41 & 23 & 19 & 10 & 12 & 46 & 13 \\
\hline 33 & 38 & 2 & 15 & 5 & 4 & 11 \\
\hline 1 & 18 & 21 & 27 & 6 & 31 & 20 \\
\hline$\infty$ & 48 & 32 & 40 & 16 & 8 & . \\
\hline 0 & 1 & 2 & \begin{tabular}{|l|}
3 \\
\end{tabular} & & 5 & 8 \\
\hline
\end{tabular}

Например, здесь записаны формулы $A^{15}=3\left[1+2, A, A^{41}=3, A, A^{24}=-1\right.$. Знак “ $\infty$ ” означает элемент 0 поля (не имеюший вида $A^{k}$ ).

ЗАмечАниЕ. Утверждение, что $A^{k} A^{l}=A^{k+l}$, очевидно, но наши рассуждения доставляют гораздо менее очевидный факт: описание операции сложения в поле в терминах (логарифмической) нумерации его элементов,

$$
A^{k}+A^{l}=A^{f(k, l)},
$$

где если $k$ стоит в клетке $\left(p_{k}, q_{k}\right)$ таблицы и $l$ - в клетке $\left(p_{l}, q_{l}\right)$, то $f(k, l)$ означает номер, стояший в таблице в клетке $\left(p_{k}+p_{l}, q_{k}+q_{l}\right)$.

ПримеР. $A^{15}+A^{41}=A^{29}, A^{14}=-A^{9}$ (так как $A^{24}=-1$ ). 


\section{§. Эргодические свойства таблиц полей Галуа}

Глядя на таблицы, вроде вьписанной вьше для $p^{a}=49$, получаешш ошушение хаотичности или "случайности" расстановки номеров в клетках таблицы.

Я предполагаю, что этому ощущению можно придать смысл точного утверждения, допускающего если и не немедленное доказательство, то, во всяком случае, численную проверку, хотя бы путем компютерного эксперимента.

Чтобы это выполнить, нужно охарактеризовать "хаотичность" каким-либо численным критерием. Я предложу ниже, не претендуя на окончательньй выбор, несколько таких критериев (вдохновляясь иногда теорией информации, а иногда физическими и астрономическими исследованиями "хаотичности" и "случайности" таких объектов, как, например, галактики и даже скопления галактик в крупномасштабной структуре Вселенной).

А) Гипотеза о равномерном распределении по площади таблицы.

Разумеется, все $n=p^{2}$ (в общем случае $p^{a}$ ) элементов поля заполняют по разу все клеточки таблицы, поэтому число элементов в любой области (состоящей из целых клеточек) пропорционально объему (площади) этой области.

Однако если вместо всех $n=p^{a}$ элементов поля рассмотреть только первые $m<n$ элементов $\left(A, A^{2}, \ldots, A^{m}\right)$, то они заполнят не все клеточки, а только $m$ из них, и тогда можно посмотреть, сколько из них попадет в данную область.

Например, мы можем рассмотреть область (скажем, с гладкой границей) $G$ на евклидовом квадрате плошади 1 , а затем поле из большого числа элементов $n=p^{2}$ и выбрать, скажем, первые $m=\theta_{n}$ элементов поля, где $0<\theta<1$.

Гипотеза состоит в том, что при $p \rightarrow \infty$ число $\mathscr{N}(G, p)$ элементов $A^{k}, k \leqslant m$, попавших в лежашие в области $G$ клеточки таблишы поля из $p^{2}$ элементов, будет асимптотически пропорционально площади этой области:

$$
\lim _{p \rightarrow \infty} \frac{\mathscr{N}(G, p)}{\theta p^{2}}=\operatorname{mes} G .
$$

В этом исследовании, разумеется, выбор $m=\theta n$ можно заменить другими выборами, рассматривая даже случай большего, но фиксированного (не зависящего от $p$ ) числа $m$, или изучая характер зависимости указанного вьше предельного перехода от параметра $\theta$.

Разумеется, предположение $a=2$ было сделано выше только для того, чтобы возможно упростить формулировку: при других значениях $a$ (включая даже $a=1$ ) задача столь же интересна.

Для численного исследования можно рассматривать, в качестве примера, специальные области $G$, например, выделенные условиями

$$
\{u[1+v \mid 0<u<p / 2\} \text { или }\{u[1+v, A, 0<u<v\}
$$

в квадрате $\left(\mathbb{Z}_{p}\right)^{2}$.

Вместо условия гладкости границы области $G$ можно было бы наложить условие ее достаточно простого алгоритмического описания (что позволило бы включить, например, "области", заданные условиями "число $u$ четно"). 


\section{В) Гипотеза о случайности переходов.}

Предположим, что вся таблица разбита на две части, I и II (скажем, область $G$, рассматривавшаяся выше, и ее дополнение).

Рассматривая последовательность точек $A^{k}, k=1,2, \ldots, m$, можно выделить четыре случая:
1) $A^{k} \in \mathrm{I}, \quad A^{k+1} \in \mathrm{I}$;
2) $A^{k} \in \mathrm{I}, \quad A^{k+1} \in \mathrm{II}$;
3) $A^{k} \in \mathrm{II}, \quad A^{k+1} \in \mathrm{I}$;
4) $A^{k} \in \mathrm{II}, \quad A^{k+1} \in \mathrm{II}$.

Естественная "гипотеза хаотичности" состоит здесь в том, что числа значений $k$ этих четырех типов пропорциональныпроизведениям вероятностей событий, т.е. если mes $I=q$, mes $I I=r$ (где $q+r=1$ ), то для чисел $N_{j}$ значений $k$ типа $j=1, \ldots, 4$ ожидаются следующие асимптотики:

$$
\lim _{p \rightarrow \infty} \frac{\mathscr{N}_{j}}{m}=\left(q^{2}, q r, r q, r^{2}\right)
$$

Эта асимптотика интересна даже для всей последовательности элементов поля, выбирая $m=n=p^{2}$ (или $p^{a}$ в случае поля из $p^{a}$ элементов).

Можно также рассматривать более длинные цепочки $\left(A^{k}, A^{k+1}, \ldots, A^{k+s}\right)$, где также можно предполагать аналогичное асимптотическое перемешивание.

Я не уверен, что эти вопросы достаточно исследованы даже и в аналогичной теории обычных динамических систем, где нет параметра $p$, но рассматриваются асимптотики все более длинных отрезков траектории системы.

\section{С) Гипотеза о случайности орбиты.}

Рассмотрим первые $m$ точек поля, $A^{k}, k=1,2, \ldots, m$, как подмножество в целочисленном квадрате $\left(\mathbb{Z}_{p}\right)^{2}$ (или в евклидовом квадрате $[0,1]^{2}$, или в $a$-мерном кубе $\left(\mathbb{Z}_{p}\right)^{a}$ или $[0,1]^{a}$, или торе $\left.\mathbb{R}^{a} / \mathbb{Z}^{a}\right)$.

Спрашивается, насколько случайно это множество - насколько его статистические характеристики отличаются от стохастических характеристик, например, случайно и независимо друг от друга набросанных в то же пространство $m$ точек?

В качестве стохастических характеристик множества точек можно рассмотреть, например, следуюшие числа:

a) минимальное расстояние между двумя точками множества;

b) максимальный радиус шара, не содержащего точек множества;

c) сумма квадратов расстояний от каждой точки множества до всех остальных (или только до ближайшей точки);

d) перколящионный радиус.

Последнее число определяется так. Если положительное число $\rho$ достаточно мало, то шары радиуса $\rho$ с центрами в точках нашего множества, если и пересекаются, образуют все же небольшие кластеры.

Напротив, при достаточно большом значении $\rho$ по цепочкам последовательно пересекаюшихся шаров можно уйти далеко. 
То значение радиуса $\rho$, при котором происходит переход от кластеров к длинным цепям (например, соединяющим противоположные стороны квадрата) назьвается перколящионньм параметром ("перколяция" - это “просачивание"), и, интерпретируя шары как дефекты оболочки сосуда, мы определяем размер и плотность дефектов, вызывающих нарушение его герметичности.

Разумеется, перколящионный радиус сильно зависит от систематичности расположения центров. Например, если центры лежат на кривой (в трехмерном кубе радиуса 1), то перколящионный радиус будет, вообще говоря, порядка $1 / m$, а если центры образуют кристаллическую решетку, то радиус гораздо больше $(1 / \sqrt[3]{m})$.

Сравнение асимптотики перколяционного радиуса орбиты $\left\{A^{k}, 1 \leqslant k \leqslant m\right\}$ с перколящионньм радиусом системы $m$ случайно набросанных в $[0,1]^{a}$ точек (скажем, при пуассоновском их распределении) показьвает степень хаотичности орбиты.

Разумеется, кроме описанных выше численных критериев стохастичности возможны и многие другие, так что задача состоит здесь более всего в том, чтобы выбрать разумньй критерий, а не только проэкспериментировать с его вьчислением: здесь важна простота вычисления критерия. Предложу еще один вариант.

D) Вариация таблицы.

Эта величина измеряет степень различия номеров $k$, заполняющих близкие клетки таблицы. Например, в случае квадратной таблищы из $p^{2}$ элементов (или из $p^{2}-1$ вычета по модулю $\left.p^{2}-1\right)$ можно рассмотреть сумму всех расстояний между соседними в таблице номерами (исключая номер $\infty$ ). Разумеется, расстояние между вычетами $a$ и $b$ по модулю $M$ - это меньшее из чисел $|a-b|$ и $M-|a-b|$ (скажем, расстояние между вычетами 1 и 6 по модулю 7 равно 2). Сумма всех $\left[4\left(p^{2}-1\right)-8\right] / 2$ расстояний в таблице характеризует хаотичность ее заполнения $p^{2}-1$ вычетом (сравнивать со случайным заполнением).

\section{§3. Проективное многообразие поля Галуа}

Проективизация векторного пространства $\left(\mathbb{Z}_{p}\right)^{a}$ определяется как многообразие прямых, проходящих через точку 0 этого пространства:

$$
P^{a-1}\left(\mathbb{Z}_{p}\right)=\left(\left(\mathbb{Z}_{p}\right)^{a} \backslash 0\right) /\left(\mathbb{Z}_{p} \backslash 0\right) .
$$

ПРИмеР. При $a=2, p=7$ мы получаем из 49 точек $u 1+v A$ плоскости $\mathbb{Z}_{7}^{2}$ восемь прямых

$$
\{(u, v): v=\lambda u\}, \quad \text { где } \lambda=0,1,2, \ldots, 6, \infty .
$$

Уравнение $v=\infty u$ означает прямую $u=0$, так что проективная прямая $P^{1}\left(\mathbb{Z}_{p}\right)$ состоит из $p+1$ точки; $p$ из них составляют аффинную прямую $(\lambda=0,1, \ldots, 6$ в нашем примере), а последняя точка назьвается бесконечно-удаленной и обозначается символом $\lambda=\infty$.

Все эти точки равноправны, и в окрестности точки $\lambda=\infty$ за координату можно взять, например, вычет $\mu=1 / \lambda$, через которьй уравнение прямой записьвается в виде $\{(u, v): u=\mu v\}$.

На многообразии $P^{a-1}$ действует, переставляя его точки, группа проективных преобразований: это спроектированные из пространства $\left(\mathbb{Z}_{p}\right)^{a}$ на $P^{a-1}$ линейные преобразования. 
Например, при $a=2$ эти преобразования проективной прямой из $p+1$ точки имеют вид

$$
\lambda \mapsto \frac{\alpha \lambda+\beta}{\gamma \lambda+\delta},
$$

где $\left(\begin{array}{ll}\alpha & \beta \\ \gamma & \delta\end{array}\right)$ - матрица линейного преобразования плоскости $\left(\mathbb{Z}_{p}\right)^{2}$, т.е. $(\alpha, \beta, \gamma, \delta)-$ вычеты по модулю $p$.

Эти преобразования переставляют точки проективного пространства $P^{a-1}$ специальньм образом: возникающая подгруппа обычно гораздо меньше всей групшы перестановок из $p+1$ элемента проективной прямой $P^{1}$ в случае $a=2$. Например, для $p=7$ число всех перестановок 8 элементов равно $8 !=56 \cdot 720$, а число проективных перестановок не превосходит $\left(7^{4}-1\right) /(7-1)=400$.

Применим эти конструкции к полю из $p^{2}$ элементов. Умножение на элемент поля действует на плоскости $\mathbb{Z}_{p}^{2}$ как линейное преобразование.

Прямые, проходящие через точку 0 поля, определены структурой поля однозначно, независимо от выбора той мультипликативной образующей, при помоши которой мы реализовали поле как плоскость $\mathbb{Z}_{p}^{2}$. Действительно, такая прямая состоит из $p$ элементов вида $\{B, B+B, B+B+B, \ldots\}$.

ТеоремА. Преобразование Фробениуса $\Phi_{k}: F \rightarrow F$, заданное возведением в степень $k$,

$$
\Phi_{k}(x)=x^{k} \quad \text { для всех } x \text { из } F
$$

(где $k$ взаимно просто с р для поля $F$ из $p^{2}$ элементов) действует на проективную прямую $P^{1}\left(\mathbb{Z}_{p}\right)$ как некоторое отображение $P \Phi_{k}$ точек этой проективной прямой. Чтобы әто отображсние было перестановкой, $k$ долэкно быть взаимно просто с $p^{a}-1$.

ДокАЗАТЕЛЬСТво. Согласно определению (2),

$$
\Phi_{k}(c x)=c^{k} \Phi_{k}(x) \text { для } c \text { из } \mathbb{Z}_{p},
$$

поэтому образы точек $x$ и $c x$ при отображении $\Phi$ робениуса $\Phi_{k}$ лежат на одной проходящей через нуль прямой пространства $\left(\mathbb{Z}_{p}\right)^{a}$ (что доказывает и теорему о действии преобразования $\Phi$ робениуса в поле из $p^{a}$ элементов на множестве $\left.P^{a-1}\left(\mathbb{Z}_{p}\right)\right)$.

ПримеР. Проективная прямая $P^{1}\left(\mathbb{Z}_{7}\right)$ состоит из восьми точек. Каждая из этих точек - прямая, состоящая из нуля и еще шести точек, а именно:

$$
\begin{array}{ll}
\lambda=0: & \{48,32,40,16,8,24\} ; \\
\lambda=1: & \{18,2,10,34,26,42\} ; \\
\lambda=2: & \{38,22,30,6,46,14\} ; \\
\lambda=3: & \{23,7,15,39,31,47\} ; \\
\lambda=4: & \{37,21,29,5,45,13\} ; \\
\lambda=5: & \{35,19,27,3,43,11\} ; \\
\lambda=6: & \{44,28,36,12,4,20\} ; \\
\lambda=\infty: & \{1,33,41,17,9,25\} .
\end{array}
$$


Элементы поля, отличные от нуля, обозначены здесь, как в таблице поля, своими логарифмами. Например, число 48 означает матрицу

$$
A^{48}=1=\left(\begin{array}{ll}
1 & 0 \\
0 & 1
\end{array}\right),
$$

число 1 означает матрицу

$$
A^{1}=A=\left(\begin{array}{ll}
0 & 2 \\
1 & 2
\end{array}\right),
$$

число 24 означает матрицу

$$
A^{24}=\left(\begin{array}{ll}
6 & 0 \\
0 & 6
\end{array}\right)=-1 \quad(\bmod 7) .
$$

ТЕОРемА. Структура проективной прямой, определенная координатой $\lambda$ на множестве $P^{1}\left(\mathbb{Z}_{p}\right)$ при помощи мультипликативной образующей $A$ поля из $p^{2}$ элементов, совпадает со структурой проективной прямой, определенной на том же множестве при помощи любой другой мультипликативной образующей, $\widetilde{A}=A^{k}$ (әде вычет числа $k$ по модулю $p^{2}-1$ взаимно прост с этим модулем).

ДокАЗАТЕльство. Как и любой элемент поля, образующая $A$ выражается через $\widetilde{A}$ как точка на плоскости, порожденной элементами 1 и $\widetilde{A}$ :

$$
A=\xi 1+\eta \widetilde{A}, \quad \text { где } \xi \in \mathbb{Z}_{p}, \quad \eta \in \mathbb{Z}_{p} .
$$

Предположим, что (ненулевой) элемент $x$ поля лежит на прямой, соответствующей данному номеру $\lambda$ (на плоскости, порожденной элементами 1 и $A$ ):

$$
x=u[1+v, \quad \text { где } \quad v=\lambda u .
$$

Тогда выражение этого элемента $x$ через 1 и $\widetilde{A}$ имеет вид

$$
x=\widetilde{u} 1+\widetilde{v} \widetilde{A}, \quad \text { где } \widetilde{u}=u+v \xi, \quad \widetilde{v}=v \eta .
$$

Эта точка лежит на прямой $\widetilde{v}=\widetilde{\lambda} \widetilde{u}$ плоскости $\langle 1, \widetilde{A}\rangle$, где

$$
\widetilde{\lambda}=\frac{v \eta}{u+v \xi}=\frac{\lambda \eta}{1+\lambda \xi} \text {. }
$$

Мы получили дробно-линейное выражение координаты $\tilde{\lambda}$ в построенной по мультипликативной образующей $\widetilde{A}$ системе координат через координату $\lambda$ в построенной по мультипликативной образующей $A$ системе.

Эта дробно-линейность и означает независимость заданной этим выбором структуры проективной прямой на множестве $P^{1}\left(\mathbb{Z}_{p}\right)$ от выбора мультипликативной образующей.

Аналогичная теорема верна и для проективного многообразия $P^{a-1}\left(\mathbb{Z}_{p}\right)$ и доказывается теми же рассуждениями. 
ПримеР. Для поля из 49 элементов и для числа $k=5$ мы находим из предыдущих таблиц для каждого значения $\lambda$ элемент $x$ соответствуюшей прямой, его выражение $\widetilde{x}$ через 1 и $\widetilde{A}=\bar{A}^{5}$, а затем номер $\widetilde{\lambda}$ проходящей через $\widetilde{x}$ прямой на плоскости, порожденной 1 и $\widetilde{A}$ (опять из приведенных таблиц):

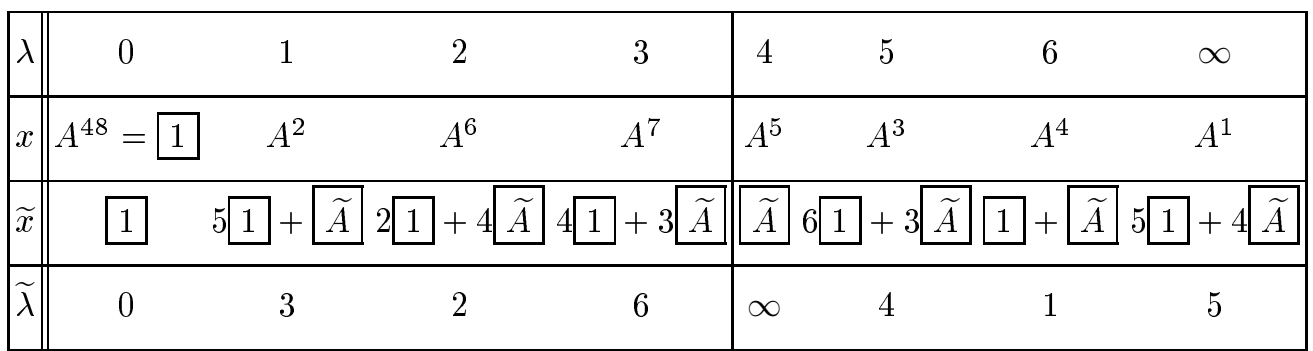

В этом примере $\widetilde{\lambda}=\frac{\lambda}{3 \lambda+2}$. Это не противоречит значениям $\xi=5, \eta=4$ (указанным в последнем столбце таблицы), так как по модулю 7

$$
\frac{\lambda}{3 \lambda+2} \equiv \frac{4 \lambda}{1+5 \lambda} \quad(\text { поскольку } 3 \cdot 4 \equiv 5,2 \cdot 4 \equiv 1)
$$

Выбирая в качестве мультипликативной образуюшей $\widetilde{A}=A^{7}$ или $A^{11}$, мы получили бы, соответственно, значения проективных координат на $P^{1}\left(\mathbb{Z}_{7}\right)$, равные

$$
\lambda_{7}=-\frac{\lambda}{2 \lambda+1}, \quad \lambda_{11}=\frac{\lambda}{\lambda+2}
$$

Поэтому четыре изученные нами проективные координаты на $P^{1}\left(\mathbb{Z}_{7}\right)$ связаны друг с другом так:

\begin{tabular}{|c||cccc|cccc|}
\hline$\lambda$ & 0 & 1 & 2 & 3 & 4 & 5 & 6 & $\infty$ \\
\hline$\lambda_{5}$ & 0 & 3 & 2 & 6 & $\infty$ & 4 & 1 & 5 \\
\hline$\lambda_{7}$ & 0 & 2 & 1 & $\infty$ & 5 & 4 & 6 & 3 \\
\hline$\lambda_{11}$ & 0 & 5 & 4 & 2 & 3 & $\infty$ & 6 & 1 \\
\hline
\end{tabular}

\section{§. Преобразования Фробениуса и их проективизации}

Определенные выше формулой (2) преобразования число $k$ взаимно просто с $p^{a}-1$ для поля из $p^{a}$ элементов) образуют коммутативную группу, элементы которой действуют на множестве точек проективной прямой $P^{1}\left(\mathbb{Z}_{p}\right)$ при $a=2$ как некоторые перестановки $P \Phi_{k}$ ее $p+1$ точек (по предыдущей теореме). 
Исследуем эти группы $\left\{\Phi_{k}\right\}$ и $\left\{P \Phi_{k}\right\}$. Обозначим через Г мультипликативную группу (Эйлера), образованную $\varphi(n)$ взаимно простыми с $n=p^{2}-1$ вычетами по модулю $n$ (для поля из 49 элементов группа $\Gamma=\mathbb{Z}_{4} \times \mathbb{Z}_{2} \times \mathbb{Z}_{2}$ состоит из 16 вьчетов

$$
\{1,5,7,11,13,17,19,23,25,29,31,35,37,41,43,47\}
$$

причем в качестве образующих трех описанных сомножителей можно взять, например, вычеты 5,7 и 17).

Описанные выше конструкции определяют гомоморфизмы коммутативных груп,

$$
\Gamma \stackrel{\varphi}{\longrightarrow}\left\{\Phi_{k}\right\} \stackrel{\psi}{\longrightarrow}\left\{P \Phi_{k}\right\}
$$

которые мы сейчас вычислим для поля из 49 элементов.

Действия преобразований лище точек прямых. Например, $\Phi_{5}(A)=A^{5}$, поэтому на прямые преобразование $\Phi_{5}$ действует так:

$$
\Phi_{5}\left(\{1,33, \ldots\} \in P^{1}\right)=\left(\{5,45, \ldots\} \in P^{1}\right) .
$$

Итак, $P \Phi_{5}(\{\lambda=\infty\})=\{\lambda=4\}$.

Такие же вычисления для других значений $\lambda$ показьвают, что преобразование $\Phi$ робениуса $P \Phi_{5}$ осуществляет следующую перестановку восьми точек:

\begin{tabular}{|c||cccc|cccc|}
\hline$\lambda(x)$ & 0 & 1 & 2 & 3 & 4 & 5 & 6 & $\infty$ \\
\hline$\lambda\left(\Phi_{5}(x)\right)$ & 0 & 1 & 2 & 5 & $\infty$ & 3 & 6 & 4 \\
\hline
\end{tabular}

Иными словами, четыре точки $\lambda=0,1,2$ и 6 остаются на месте, а члены пар $(3,5)$ и $(4, \infty)$ переставляются внутри пары.

Из этого следует, что перестановка $\Phi_{5}$ не является проективным преобразованием (иначе, оставляя на месте четыре точки, она не сдвигала бы ни одной).

Аналогичные выгисления для всех 16 преобразований Фробениуса $\Phi_{k}(k \in \Gamma)$ показывают, что они осушествляют всего четыре разных перестановок восьми точек проективной прямой, а именно $P \Phi_{k}$ переводит точку $x$ с координатой $\lambda(x)=0,1, \ldots, 6, \infty$ в точку, координата $\lambda$ которой задана следующей таблицей:

\begin{tabular}{|cccc||cccc|cccc|}
\hline \multicolumn{4}{|c||}{$k$} & \multicolumn{1}{|c|}{$\lambda\left(P \Phi_{k}(x)\right)$} \\
\hline 1, & 17, & 25, & 41 & 0 & 1 & 2 & 3 & 4 & 5 & 6 & $\infty$ \\
\hline 5, & 13, & 29, & 37 & 0 & 1 & 2 & 5 & $\infty$ & 3 & 6 & 4 \\
\hline 7, & 23, & 31, & 47 & 0 & 2 & 1 & $\infty$ & 5 & 4 & 6 & 3 \\
\hline 11, & 19, & 35, & 43 & 0 & 2 & 1 & 4 & 3 & $\infty$ & 6 & 5 \\
\hline
\end{tabular}


Итак, все эти четыре перестановки являются инволюциями (их квадраты - тождественные преобразования), подобными притом отражениям следуюших фигур в горизонтальной оси симметрии:
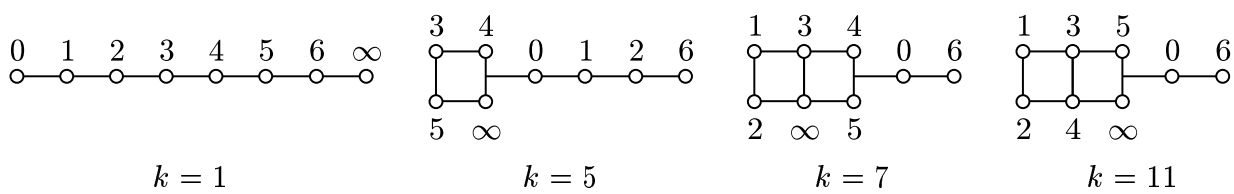

Преобразования $P \Phi_{1}=1$ и $P \Phi_{7}$ проективные:

$$
\lambda\left(P \Phi_{1}(x)\right) \equiv \lambda(x), \quad \lambda\left(P \Phi_{7}(x)\right)=\frac{-\lambda(x)}{2 \lambda(x)+1}
$$

Преобразования $P \Phi_{5}$ и $P \Phi_{11}$ проективными не являются (они не сохраняют описанную выше проективную структуру на $P^{1}\left(\mathbb{Z}_{7}\right)$, а переводят ее в другую). При этом оба они переводят ее в одну и ту же другую структуру, поскольку $\Phi_{5} \Phi_{7}=\Phi_{35}$ и $P \Phi_{35}=P \Phi_{11}$, так что $P \Phi_{11}=\left(P \Phi_{5}\right)\left(P \Phi_{7}\right)$, а $P \Phi_{7}$ сохраняет стандартную структуру. Эта стандартная структура переходит под действием перестановок $P \Phi_{5}$ и $P \Phi_{11}$ в одну и ту же нестандартную проективную структуру на $P^{1}\left(\mathbb{Z}_{7}\right)$.

Это же рассуждение показьвает, что преобразование $P \Phi_{7}$ сохраняет и нестандартную проективную структуру, описанную вьше.

Из всего этого следует, что группа преобразований $\Phi$ робениуса $\left\{\Phi_{k}\right\}$ является изоморфным образом групш Эйлера $Г$ при гомоморфизме $\varphi$, отображающем вычет $k$ в преобразование $\Phi_{k}$. Гомоморфизм проективизации $\psi:\left\{\Phi_{k}\right\} \rightarrow\left\{P \Phi_{k}\right\}$ отображает этот образ на групу $\mathbb{Z}_{2}^{2}$ из четырех вьписанных вьше перестановок.

Ядро этого гомоморфизма состоит из четырех преобразований $\left\{\Phi_{1}, \Phi_{17}, \Phi_{25}, \Phi_{41}\right\}$, также образуюших групу $\mathbb{Z}_{2}^{2}$. В отличие от своих проективизаций $P \Phi_{k}$, эти преобразования, кроме $\Phi_{1}$, не оставляют все элементы поля на местах, так как, например,

$$
\Phi_{17}\left(A^{k}\right)=4^{k} A^{k}, \quad \Phi_{25}\left(A^{k}\right)=6^{k} A^{k}, \quad \Phi_{41}\left(A^{k}\right)=3^{k} A^{k} .
$$

Сохранение проективной структуры преобразованием $\Phi_{7}$ объясняется тем, что это преобразование является автоморфизмом поля Галуа: для этого взаимно однозначного отображения $f$ поля в себя при любых $x$ и $y$

$$
f(x+y)=f(x)+f(y), \quad f(x y)=f(x) f(y) .
$$

Все автоморфизмы поля из $p^{a}$ элементов образуют циклическую групу $\mathbb{Z}_{a}$ из $a$ элементов, образуюшей является автоморфизм $\Phi$ робениуса $\Phi_{p}$.

Например, группа автоморфизмов поля $\mathbb{Z}_{p}$ вьчетов по модулю $p$ состоит из одного лишь тождественного преобразования. Групу автоморфизмов поля из 49 элементов легко вычислить так. Автоморфизм обязан быть преобразованием Фробениуса, поскольку он сохраняет операцию умножения. Но из операций Фробениуса $\Phi_{k}$ сложение сохраняют только $\Phi_{1}$ и $\Phi_{7}$. Сравнение $(x+y)^{7} \equiv x^{7}+y^{7}$ следует из делимости на 7 остальных биномиальных коэффициентов $C_{7}^{u}$. 
Группа автоморфизмов поля из 49 элементов состоит поэтому из двух элементов: это $\mathbb{Z}_{2}=\{1, f\}$, где $f(x) \equiv x^{7}$. При этом $f^{2}(x)=f(f(x)) \equiv\left(x^{7}\right)^{7}=x^{49} \equiv x$, так что $f^{2}=1$.

Действие преобразований $\Phi$ робениуса и их проективных вариантов $P \Phi_{k}$ на проективные структуры многообразий $P^{a-1}\left(\mathbb{Z}_{p}\right)$ для полей из $p^{a}>49$ элементов, даже в случае $a=2$, заслуживает, по моему мнению, изучения, аналогичного проведенному вьше для $p^{a}=49$.

Можно даже надеяться на связь этих вопросов со сравнениями для биномиальных и мультиномиальных коэффициентов, обобшаюшими малую теорему Ферма и ее доказанньй Эйлером вариант на матрицы с целыми элементами. Гипотетическое обобщение сравнения Эйлера-Ферма имеет вид

$$
\operatorname{tr} A^{n} \equiv \operatorname{tr} A^{n-\varphi(n)} \quad\left(\bmod \left(n=p^{a}\right)\right) .
$$

Это сравнение доказано сейчас в [1] для $a=1,2$ и 3 , а для $a=4$ оно доказано при $p \leqslant 29$ (причем, повидимому, это доказательство указывает на то, что вопрос о справедливости сравнения (3) для всех целочисленных матрищ $A$ (любого конечного порядка) алгоритмически разрешим, по меньшей мере для каждого фиксированного простого числа $p$, при $a=4)$.

Однако, например, для $n=6$ сравнение (3) неверно - это и подсказьвает возможность его связи с полями Галуа.

Сравнения для биномиальных коэффищиентов, упомянутые вьше, начинаются с исследования удивительной функции $x(a, b)$, заданной уравнением

$$
C_{p a}^{p b}-C_{a}^{b}=p^{x(a, b)} z, \quad \text { где }(z, p)=1 .
$$

Например, для $a=4, b=2$ разность биномиальных коэффищиентов делится на удивительно большую степень простого числа $p=2$ :

$$
C_{8}^{4}-C_{4}^{2}=70-6=2^{6} .
$$

Функция $x(a, b)$, определенная соотношением (4), сама обладает удивительной двоякой $p$-периодичностью, нарушаемой после $p$ повторений, но вся эта картина обладает двоякой $p^{2}$-периодичностью, нарушаемой лишь в масштабе $p^{3}$, и так далее. $\mathrm{K}$ сожалению, эта своеобразная $p$-модулярность остается чисто экспериментальным компютерным наблюдением, не связанньм пока ни с эллиптическими функциями, подсказываемыми двоякой периодичностью, ни с полями Галуа, на роль которых указьвает появление $p^{a}$. Можно только надеяться, что привлечение полей Галуа может пролить свет на эти эмпирические сравнения, чем и объясняется появление настоящей статьи.

\section{$\S 5$. Проективные структуры поля Галуа из $p^{2}$ элементов, где $p=11$}

Для $p=11$ в качестве мультипликативной образующей можно взять матрицу $A=\left(\begin{array}{ll}0 & 3 \\ 1 & 1\end{array}\right)$. Элементы поля представляются тогда 121 матрицей $\left\{0, A^{k}\right\}$, где $1 \leqslant$ 
$k \leqslant 120$, из вычетов по модулю 11 (причем $A^{120}=1$ ); операшии поля - сложение и умножение матриц. Запишем эти матрицы в виде

$$
A^{k}=u_{k} 1+v_{k} A
$$

(где $\left.1=A^{120}, A=A^{1}\right)$. Числа (точнее, вычеты по модулю $\left.p\right) u_{k}$ и $v_{k}$ определяют следующую таблищу логарифмов (заполнение “тора" $\left(\mathbb{Z}_{p}\right)^{2}$ числами $k$, изображаюшими матрицы $A^{k}$, и символом $\infty$, изображающим нулевую матрицу) из псевдослучайных чисел (точнее, вычетов по модулю $n=p^{2}-1=120$ ):

\begin{tabular}{|c|c|c|c|c|c|c|c|c|c|c|c|}
\hline $10 \mathrm{~A}$ & 61 & 11 & 15 & 76 & 22 & 43 & 78 & 53 & 62 & 56 & 105 \\
\hline $9 A$ & 25 & 42 & 95 & 17 & 99 & 26 & 40 & 20 & 106 & 69 & 7 \\
\hline $8 A$ & 13 & 94 & 14 & 83 & 115 & 8 & 87 & 30 & 57 & 28 & 5 \\
\hline $7 A$ & 109 & 4 & 6 & 104 & 59 & 70 & 101 & 33 & 63 & 91 & 110 \\
\hline $6 A$ & 37 & 32 & 29 & 19 & 52 & 107 & 81 & 38 & 54 & 118 & 111 \\
\hline $5 A$ & 97 & 51 & 58 & 114 & 98 & 21 & 47 & 112 & 79 & 89 & 92 \\
\hline $4 A$ & 49 & 50 & 31 & 3 & 93 & 41 & 10 & 119 & 44 & 66 & 64 \\
\hline $3 A$ & 73 & 65 & 88 & 117 & 90 & 27 & 68 & 55 & 23 & 74 & 34 \\
\hline $2 A$ & 85 & 67 & 9 & 46 & 80 & 100 & 86 & 39 & 77 & 35 & 102 \\
\hline $1 A$ & 1 & 45 & 116 & 2 & 113 & 18 & 103 & 82 & 16 & 75 & 71 \\
\hline $0 A$ & $\infty$ & 120 & 84 & 72 & 48 & 96 & 36 & 108 & 12 & 24 & 60 \\
\hline$v / u$ & 0 & 1 & 2 & 3 & 4 & 5 & 6 & 7 & 8 & 9 & 10 \\
\hline
\end{tabular}

Вычисление этой таблишы поля сокрашается вдвое ее очевидной симметрией (относительно отмеченного кружочком центра):

$$
\left(u_{k+(n / 2)}, v_{k+(n / 2)}\right)+\left(u_{k}, v_{k}\right)=(p-1, p-1) .
$$

Это соотношение вытекает из того, что $A^{n / 2}=-1$.

Из приведенной вьше таблицы поля следует описание прямых $\{v=\lambda u\}$, нумеруемых афффинной координатой $\lambda$ в $P^{1}\left(\mathbb{Z}_{(p=11)}\right)$ : такая прямая образована парами $\left(u_{k}, v_{k}\right)$, где $k$ указано в следуюшей таблице

\begin{tabular}{|c||cccccccccccc|}
\hline$\lambda$ & 0 & 1 & 2 & 3 & 4 & 5 & 6 & 7 & 8 & 9 & 10 & $\infty$ \\
$k(\bmod 12)$ & 0 & 9 & 7 & 5 & 2 & 3 & 8 & 4 & 10 & 6 & 11 & 1 \\
\hline
\end{tabular}

Это описание прямых объясняется тем, что матрица $A^{p+1}\left(=A^{12}\right)$ скалярная, поэтому $(c A)^{k+(p+1)}=C A^{k}$. Число скалярных матриц $A^{m}$ равно $p-1$, поэтому для скалярной матрищы показатель $m$ делится на число $\left(p^{2}-1\right) /(p-1)=p+1^{1}$.

\footnotetext{
${ }^{1}$ Для поля из $p^{a}$ элементов прямые характеризуются остатками от деления показателей $k$ на число $\left(p^{a}-1\right) /(p-1)=p^{a-1}+\cdots+p+1$ точек проективного пространства $P^{a-1}\left(\mathbb{Z}_{p}\right)$.
} 
Из приведенных формул видно, что прямая $\{v=\lambda u\}$ переводится преобразованием $\Phi$ робениуса $\Phi_{m}$ (заданным условием $\Phi_{m}(x)=x^{m}$ для всякого элемента $x$ нашего поля) в прямую $\left\{v=\lambda_{m} u\right\}$, где $\lambda_{m}$ получается из $\lambda$ некоторой перестановкой $P \Phi_{m}: P^{1}\left(\mathbb{Z}_{p}\right) \rightarrow P^{1}\left(\mathbb{Z}_{p}\right) p+1$ точки конечной проективной прямой. А именно, алгоритм вычисления $\lambda_{m}(\lambda)$ таков:

$$
\lambda \mapsto k(\bmod (p+1)) \mapsto m k(\bmod (p+1)) \mapsto \lambda_{m}
$$

(где соответствие между $\lambda$ и $k$ указано выше).

Вычисление по этому алгоритму перестановок $P \Phi_{m}$ приводит к приведенному в следующей таблице ответу для тридщати двух $\left(=\varphi\left(120=p^{2}-1\right)\right)$ взаимно простых с $p^{3}-1$ значений,

$$
m=1,7,11,13,17,19,23,29,31,37,41,43,47,49,53,59, \ldots, 119
$$

(вместе с $m$ в списке есть и $120-m$ ).

Мультипликативная група взаимно простых с числом 120 вычетов есть

$$
\Gamma=\mathbb{Z}_{4} \times \mathbb{Z}_{2}^{3} \quad \text { (образуюшие: } m=7 \text { в } \mathbb{Z}_{4}, m=11,19,61 \text { в } \mathbb{Z}_{2}^{3} \text { ). }
$$

Она отображается на группу преобразований

Отображение проективизации, $\psi:\left\{\Phi_{m}\right\} \rightarrow\left\{P \Phi_{m}\right\}$, имеет ядро из восьми элементов,

$$
\begin{aligned}
& \operatorname{Ker} \psi=\left\{\Phi_{m}: m=1,13,37,49,61,73,97,109\right\} \approx \mathbb{Z}_{4} \times \mathbb{Z}_{2}, \\
& \mathbb{Z}_{4}:\left\{1,13,13^{2} \sim 49,13^{3} \sim 37,\left(13^{4} \sim 1\right)\right\}, \\
& \mathbb{Z}_{2}:\{1,61\} ; 61 \cdot 13 \sim 71, \quad 61 \cdot 49 \sim 109, \quad 61 \cdot 37 \sim 97 .
\end{aligned}
$$

Образ $\left\{P \Phi_{m}\right\}=\operatorname{Im} \psi$ отображения проективизации $\psi$ состоит из четырех перестановок (первая из них тождественна): $\operatorname{Im} \psi=\left\{P \Phi_{1}, P \Phi_{7}, P \Phi_{11}, P \Phi_{17}\right\} \approx \mathbb{Z}_{2}^{2}$ :

\begin{tabular}{|c||cccccccccccc|}
\hline$\lambda_{1}(x)=\lambda\left(P \Phi_{1}(x)\right)$ & 0 & 1 & 2 & 3 & 4 & 5 & 6 & 7 & 8 & 9 & 10 & $\infty$ \\
\hline$\lambda_{7}(x)=\lambda\left(P \Phi_{7}(x)\right)$ & 0 & 5 & $\infty$ & 10 & 4 & 1 & 6 & 7 & 8 & 9 & 3 & 2 \\
$\lambda_{11}(x)=\lambda\left(P \Phi_{11}(x)\right)$ & 0 & 5 & 3 & 2 & 8 & 1 & 7 & 6 & 4 & 9 & $\infty$ & 10 \\
$\lambda_{17}(x)=\lambda\left(P \Phi_{17}(x)\right)$ & 0 & 1 & 10 & $\infty$ & 8 & 5 & 7 & 6 & 4 & 9 & 2 & 3 \\
\hline
\end{tabular}

Тождественное преобразование $P \Phi_{1}$ и преобразование $P \Phi_{11}$ (доставляемое автоморфизмом $\Phi_{11}$ поля из $11^{2}$ элементов) сохраняют естественную проективную структуру $P_{1}=P_{11}$ проективной прямой, определяемую аффинной координатой $\lambda$, а преобразования $P \Phi_{7}$ и $P \Phi_{17}$ переводят ее в другую проективную структуру, $P_{7}=P_{17}$ (одну и ту же), для которой аффинной координатой является, например, функция $\lambda_{7}$, заданная тождеством $\lambda_{7}(x)=\lambda\left(P \Phi_{7}(x)\right)$, или проективно связанная с ней функция,

$$
\lambda_{17}=-\lambda_{7} /\left(\lambda_{7}+1\right) .
$$


Точно так же связаны и аффинные координаты $\lambda_{1}=\lambda$ и $\lambda_{11}=-\lambda /(\lambda+1)$ проективной структуры $P_{1}=P_{11}$.

Заметим, что “естественная" проективная структура $P_{1}$ на множестве $P^{1}\left(\mathbb{Z}_{11}\right)$ определена выбором мультипликативной образующей $A$ поля Галуа, и переход к другой образуюшей, $A^{m}$, осушествляется преобразованием $\Phi$ робениуса $\Phi_{m}$, так что все проективные структуры на множестве $P^{1}\left(\mathbb{Z}_{p}\right)$, соответствующие разньм выборам мультипликативной образующей, получаются друг из друга проективизированньми преобразованиями $\Phi$ робениуса, $P \Phi_{m}$.

При $p=11$ все 32 образуюшие $A^{m}$ порождают лишш две структуры, $P_{1}$ и $P_{7}$. Преобразование $P \Phi_{11}$ сохраняет обе проективные структуры, а преобразования $P \Phi_{7}$ и $P \Phi_{17}$ переставляют их.

§. Проективные структуры поля Галуа из $p^{2}$ элементов, где $p=13$

Для $p=13$ годится образующая матрица $A=\left(\begin{array}{ll}0 & 2 \\ 1 & 4\end{array}\right)$. Элементы поля представляются 169-ю матрицами $\left\{0, A^{k}\right\}$ из вычетов по модулю $p=13$, где $1 \leqslant k \leqslant 169$ (операции - сложение и умножение матриц). Соответствующая выбору образующей $A$ таблица поля (составленная из вычетов по модулю $p^{2}-1=168$ ) образована следуюшими псевдослучайными числами (вычетами):

\begin{tabular}{|c|c|c|c|c|c|c|c|c|c|c|c|c|c|}
\hline $12 A$ & 85 & 45 & 161 & 165 & 13 & 76 & 58 & 47 & 158 & 122 & 23 & 166 & $\$ 4$ \\
\hline 114 & 15 & 145 & 143 & 88 & 91 & 52 & 95 & 121 & 111 & 96 & 6 & 162 & 156 \\
\hline$A$ & 141 & 10 & 132 & 101 & 79 & 114 & 49 & 54 & 103 & 53 & 120 & 46 & \\
\hline $9 A$ & 113 & 51 & 75 & 41 & 73 & 26 & 18 & 104 & 21 & 92 & 150 & 86 & \\
\hline 8 & 27 & 32 & 39 & 40 & 100 & 87 & 164 & 55 & 106 & 89 & 35 & 65 & \\
\hline & 71 & 152 & 108 & 33 & 62 & 151 & 31 & 50 & $y$ & 144 & 44 & 167 & \\
\hline 6 & 155 & 63 & 83 & 128 & 60 & 93 & 134 & 115 & 67 & 146 & 117 & 24 & \\
\hline $5 A$ & 43 & 34 & 149 & 119 & 5 & 22 & 139 & 80 & $J$ & 16 & 124 & 123 & \\
\hline $4 A$ & 29 & 109 & 2 & 66 & 8 & 105 & 20 & 102 & 110 & 157 & 125 & 159 & \\
\hline 3 & 57 & 153 & 130 & 36 & 137 & 19 & 138 & 133 & 30 & 163 & 17 & 48 & \\
\hline & 99 & 72 & 78 & 90 & 12 & 27 & 37 & 11 & 136 & 7 & 4 & 59 & \\
\hline $1 A$ & 1 & 148 & 82 & 107 & 38 & 74 & 131 & 142 & 160 & 97 & 81 & 77 & \\
\hline $\overrightarrow{0 A}$ & $\infty$ & 168 & 98 & 56 & 28 & 47 & 154 & 70 & 126 & 112 & 140 & 14 & \\
\hline & & 1 & \begin{tabular}{|l|}
2 \\
\end{tabular} & 3 & \begin{tabular}{|l|}
4 \\
\end{tabular} & 5 & 6 & 7 & 8 & 9 & \begin{tabular}{|l|}
10 \\
\end{tabular} & 11 & \\
\hline
\end{tabular}

Из этой таблицы поля следует, что прямая $\{v=\lambda u\}$, соответствуюшая значению $\lambda$ аффинной координаты в $P^{1}\left(\mathbb{Z}_{13}\right)$, заполнена элементами $A^{k}$, для которых остаток от деления $k$ на $p+1$ связан с $\lambda$ таблицей

\begin{tabular}{|c||cccccccccccccc|}
\hline$\lambda$ & 0 & 1 & 2 & 3 & 4 & 5 & 6 & 7 & 8 & 9 & 10 & 11 & 12 & $\infty$ \\
$k(\bmod 14)$ & 0 & 8 & 2 & 13 & 11 & 6 & 7 & 12 & 4 & 9 & 10 & 5 & 3 & 1 \\
\hline
\end{tabular}


Взаимно простые с числом $p^{2}-1=168$ вычеты образуют мультипликативную группу $\Gamma \approx \mathbb{Z}_{6} \times \mathbb{Z}_{2}^{3}$ из 48 элементов,

$$
\begin{aligned}
m= & 1,5,11,13,17,19,23,25,29,31,37,41,43,47,53,55,59,61 \\
& 65,67,71,73,79,83,85, \ldots, 167
\end{aligned}
$$

(включающую вместе с вычетом $m$ вычет $168-m$ ). Група преобразований Фробениуса, $\left\{\Phi_{m}\right\}$, изоморфна группе вычетов $\Gamma$.

Ядро гомоморфизма проективизации $\psi:\left\{\Phi_{m}\right\} \rightarrow\left\{P \Phi_{m}\right\}$ состоит из тех восьми преобразований $\Phi$ робениуса $\Phi_{m}$, для которых $m$ сравнимо с 1 по модулю 14 (вследствие чего матрища $A^{m}$ скалярна): $m=1,29,43,71,85,113,127,155, \operatorname{Ker} \psi \approx \mathbb{Z}_{2}^{3}$. Эти восемь преобразований оставляют каждую точку проективной прямой $P^{1}\left(\mathbb{Z}_{13}\right)$ на месте.

Фактор-група $\left\{\Phi_{m}\right\} /(\operatorname{Ker} \psi) \approx \mathbb{Z}_{6} \approx \operatorname{Im} \psi\left\{P \Phi_{m}\right\}$ представлена преобразованиями $P \Phi_{m}$, где $m=1,13,5,11,17,37$.

За образующую этой циклической группы можно выбрать преобразование $P \Phi_{5}$ : остальные четыре перестановки четырнадцати точек прямой $P^{1}\left(\mathbb{Z}_{13}\right)$ даются формулами

$$
P \Phi_{11}=\left(P \Phi_{5}\right)^{2}, \quad P \Phi_{13}=\left(P \Phi_{5}\right)^{3}, \quad P \Phi_{37}=\left(P \Phi_{5}\right)^{4}, \quad P \Phi_{17}=\left(P \Phi_{5}\right)^{5} .
$$

Все эти шесть перестановок четырнадщати точек проективной прямой определяются в аффинной координате $\lambda$ на проективной прямой $P^{1}\left(\mathbb{Z}_{13}\right)$ функциями $\lambda_{m}(x)=$ $\lambda\left(P \Phi_{m}(x)\right)$, заданньми таблицей

\begin{tabular}{|c||cccccccccccccc|}
\hline$m=1$ & 0 & 1 & 2 & 3 & 4 & 5 & 6 & 7 & 8 & 9 & 10 & 11 & 12 & $\infty$ \\
$m=5$ & 0 & 7 & 10 & 9 & 3 & 2 & 6 & 8 & 5 & 12 & 1 & 4 & $\infty$ & 11 \\
$m=11$ & 0 & 8 & 1 & 12 & 9 & 10 & 6 & 5 & 2 & $\infty$ & 7 & 3 & 11 & 4 \\
$m=13$ & 0 & 5 & 7 & $\infty$ & 12 & 1 & 6 & 2 & 10 & 11 & 8 & 9 & 4 & 3 \\
$m=17$ & 0 & 10 & 5 & 4 & 11 & 8 & 6 & 1 & 7 & 3 & 2 & $\infty$ & 9 & 12 \\
$m=37$ & 0 & 2 & 8 & 11 & $\infty$ & 7 & 6 & 10 & 1 & 4 & 5 & 12 & 3 & 9 \\
\hline
\end{tabular}

Разумеется, $\left(P \Phi_{5}\right)^{6}=1,\left(P \Phi_{11}\right)^{3}=1,\left(P \Phi_{13}\right)^{2}=1,\left(P \Phi_{37}\right)^{3}=1,\left(P \Phi_{17}\right)^{6}=1$ (в группе $\mathbb{Z}_{6}$ ).

Преобразование $P \Phi_{13}$ (соответствуюшее автоморфизму $\Phi_{13}$ поля Галуа) сохраняет проективную структуру $P_{1}$, заданную на множестве $P^{1}\left(\mathbb{Z}_{13}\right)$ аффинной координатой $\lambda_{1}=\lambda$ :

$$
\lambda_{13}=-\lambda /(4 \lambda+1), \text { поэтому } P_{13}=P_{1} .
$$

Напротив, преобразования $P \Phi_{5}, P \Phi_{11}, P \Phi_{37}, P \Phi_{17}$ переводят структуру $P_{1}$ в две другие структуры, $P_{5}=P_{37}$ и $P_{11}=P_{17}$. Эти структуры получились бы, если бы за мультикативную образующую, вместо $A$, был бы выбран элемент $A^{5}$ или $A^{37}$ в первом случае и $A^{11}$ или $A^{17}$ во втором. Преобразование $P \Phi_{13}$ сохраняет и эти структуры. 
Таким образом, группа $\operatorname{Im} \psi \approx \mathbb{Z}_{6}$ действует на тройку проективных структур $\left(P_{1}, P_{5}, P_{11}\right)$, циклически переставляя их: $\left(P \Phi_{5}\right) P_{1}=P_{5},\left(P \Phi_{5}\right) P_{5}=P_{11}$, $\left(P \Phi_{5}\right) P_{11}=P_{1}$. Мы построили по полю из 169 элементов группу $\mathbb{Z}_{3}$ циклических перестановок трех проективных структур на множестве $P^{1}\left(\mathbb{Z}_{13}\right)$.

\section{СПИСОК ЛИТЕРАТУРЫ}

[1] V. I. Arnold. Fermat dynamics of matrices, finite circles and finite Lobachevsky planes // Cahiers du CEREMADE. № 0434. Paris: Université Paris-Dauphine, 3 juin 2004.

Математический институт

Поступила в редакцию им. В. А. Стеклова РАН 18.08.2004 